\title{
Direct imaging of the near field and dynamics of surface plasmon resonance on gold nanostructures using photoemission electron microscopy
}

\author{
Quan Sun $^{1,2}$, Kosei Ueno ${ }^{1,3}$, Han $\mathrm{Yu}^{1}$, Atsushi Kubo ${ }^{4}$, Yasutaka Matsuo ${ }^{1}$ and Hiroaki Misawa ${ }^{1}$
}

Localized surface plasmon resonance (LSPR) can be supported by metallic nanoparticles and engineered nanostructures. An understanding of the spatially resolved near-field properties and dynamics of LSPR is important, but remains experimentally challenging. We report experimental studies toward this aim using photoemission electron microscopy (PEEM) with high spatial resolution of sub-10 nm. Various engineered gold nanostructure arrays (such as rods, nanodisk-like particles and dimers) are investigated via PEEM using near-infrared (NIR) femtosecond laser pulses as the excitation source. When the LSPR wavelengths overlap the spectrum of the femtosecond pulses, the LSPR is efficiently excited and promotes multiphoton photoemission, which is correlated with the local intensity of the metallic nanoparticles in the near field. Thus, the local field distribution of the LSPR on different Au nanostructures can be directly explored and discussed using the PEEM images. In addition, the dynamics of the LSPR is studied by combining interferometric time-resolved pump-probe technique and PEEM. Detailed information on the oscillation and dephasing of the LSPR field can be obtained. The results identify PEEM as a powerful tool for accessing the near-field mapping and dynamic properties of plasmonic nanostructures.

Light: Science \& Applications (2013) 2, e118; doi:10.1038/lsa.2013.74; published online 20 December 2013

Keywords: femtosecond laser; local field enhancement; near-field imaging; photoemission electron microscopy; surface plasmon resonance

\section{INTRODUCTION}

Because of the rapid development of nanofabrication techniques, metallic nanostructures that can exhibit localized surface plasmon resonance (LSPR) can be fabricated using several methods. The resonance frequency and amplitude of LSPR on metallic nanostructures are known to depend on the metal materials, shapes, and surrounding media. ${ }^{1-4}$ In addition, the LSPR can confine optical fields in nanoscale space, leading to the so-called local field enhancement effect. These unique properties promote the application of LSPR in many fields, such as surface-enhanced Raman scattering, ${ }^{5-8}$ sensing, ${ }^{1,9,10}$ plasmonassisted photochemical reactions $s^{4,11,12}$ and photocurrent generation. ${ }^{13-15}$ To further understand the LSPR mechanism and to optimize the design of the plasmonic nanostructures for most applications, the near-field properties of the LSPR fields (especially the near-field distribution of the plasmonic nanostructures) must be determined. To date, investigations of the optical properties of LSPR have largely relied on far-field spectroscopic techniques or numerical simulations. Several experimental approaches have been utilized to visualize the near field, including scanning photoionization microscopy, ${ }^{16,17}$ scanning near-field optical microscopy, ${ }^{18-21}$ nonlinear luminescence or fluorescent microscopy, ${ }^{22,23}$ nonlinear photopolymerization ${ }^{24,25}$ and near-field ablation of a substrate. ${ }^{26-29}$ However, these approaches have practical limitations; specifically, both scanning photoionization microscopy and scanning near-field optical microscopy require a scanning process to acquire a near-field image, and their spatial resolution barely reaches the sub-50-nm level. Only a limited study has demonstrated sub-10 $\mathrm{nm}$ resolution in imaging metal nanogaps using a scattering-type scanning near-field optical microscopy. ${ }^{21}$ Nonlinear fluorescence microscopy and photopolymerization require special treatment of the investigated samples; the metallic nanostructures must be coated with a layer of dye material or photopolymers. This additional layer also alters the properties of the LSPR. The near-field ablation technique imposes permanent damage onto the samples. Therefore, directly imaging the near fields of plasmonic nanostructures with high spatial resolution remains an experimental challenge. Understanding the dynamic properties of the LSPR is also highly desired, but rarely realized experimentally.

The recent development of multiphoton photoemission electron microscopy (MP-PEEM) has resulted in a novel approach to directly visualize the near-field of LSPR supported on metallic nanostructures. ${ }^{30-35}$ Photoemission electron microscopy records the electrons emitted from a sample in response to the absorption of incident photons. Conventional PEEM uses ultraviolet (UV) light or X-ray radiation as the excitation source and has been demonstrated as a

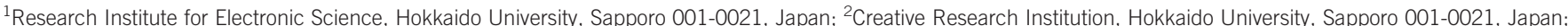
${ }^{3}$ PRESTO, Japan Science and Technology Agency, Kawaguchi 332-0012, Japan and ${ }^{4}$ Institute of Physics, University of Tsukuba, Tsukuba 305-8571, Japan

Correspondence: Professor H Misawa, Research Institute for Electronic Science, Hokkaido University, Sapporo 001-0021, Japan

E-mail: misawa@es.hokudai.ac.jp

Received 11 March 2013; revised 12 June 2013; accepted 16 August 2013 
powerful imaging and characterization tool in the fields of surface physics/chemistry, material growth and magnetic materials. ${ }^{36,37}$ In contrast to scanning electron microscopy, PEEM can directly image surface areas emitting photoelectrons in real time without scanning. MP-PEEM is based on the multiphoton photoemission from a species excited by ultrashort (picosecond or femtosecond) laser pulses. The probability of multiphoton excitation is typically low; however, LSPRassisted local field enhancement can promote the multiphoton excitation of metallic nanostructures and render MP-PEEM suitable for direct imaging of the near-field LSPR. The combination of MPPEEM with pump-probe techniques, namely, time-resolved MPPEEM (TR-MP-PEEM), ${ }^{32,38,39}$ was established to investigate the dynamics of LSPR and the propagating surface plasmon polariton. Two-photon PEEM investigations have typically been performed on Ag nanostructures excited by near-ultraviolet femtosecond laser pulses ( $\sim 400 \mathrm{~nm}$, double frequency of a Ti:sapphire laser). ${ }^{30-32}$ The LSPR of the Au nanoparticles lies in visible region $(\sim 510 \mathrm{~nm})$ and near-infrared (NIR) region so that it cannot be excited by near-ultraviolet light. For example, the LSPR wavelength of the Au nanostructures studied in this paper is in the range of 700-900 nm, which is far from the double frequency of Ti:sapphire laser pulses. Therefore, the fundamental frequency of Ti:sapphire femtosecond laser pulses should be more suitable for exciting such LSPR modes. However, only few reports have described investigations of the LSPR using MP-PEEM with femtosecond pulses from an 800-nm excitation source, ${ }^{33-35}$ likely because of the difficulty in achieving higher nonlinear photoemission. Furthermore, no reports have yet described the direct observation of the dynamics of LSPR on Au nanoparticles using TR-MP-PEEM at this wavelength.

In this study, we established an MP-PEEM system using NIR ultrashort femtosecond laser pulses as the excitation source. We used the system to directly image the near field of LSPR on various Au nanoparticle arrays. The wide bandwidth (ranging from 650-1050 nm) of the laser pulses allowed excitation of the LSPR over a wide spectral region under resonance or near-resonance conditions. The near-field mapping and the local field enhancement sites can be precisely obtained because of the high spatial resolution of PEEM $(\sim 8 \mathrm{~nm})$, which enable us to visualize polarization distribution in a metal nanostructure or an aggregate of nanostructures. It is worth mentioning that in the most case of plasmon imaging by PEEM, the spatial resolution has been limited to $\sim 50 \mathrm{~nm} .^{30,32,33,35,38}$ The ultrashort pulse duration (7 fs) makes this light source suitable for time-resolved studies. We also combined MP-PEEM with an interferometric time-resolved photoemission technique to establish a TR-MP-PEEM system to investigate the dynamics of the LSPR. This paper is organized as follows. First, we report the near-field mapping of three different $\mathrm{Au}$ nanoparticle arrays via MP-PEEM. For complicated structures in particular (using dimers as an example), the local field enhancement sites can be directly visualized by simultaneous irradiation with NIR femtosecond pulses and UV light. Second, we present dynamics studies of the LSPR via TR-MP-PEEM. The oscillation and dephasing of the LSPR fields in the NIR region on the Au nanoparticles were observed using this time-resolved technique for the first time. In particular, we demonstrated the ability of the TR-MP-PEEM technique to resolve two different LSPR frequencies.

\section{MATERIALS AND METHODS}

To fabricate the gold nanoparticle arrays, we applied electron-beam lithography followed by metal sputtering and lift-off techniques. The fabrication procedure used in this study was similar to the process described in our earlier reports. ${ }^{22,25}$ Patterns of $\mathrm{Au}$ nanostructures were formed on $\mathrm{Nb}$-doped $\mathrm{TiO}_{2}$ (110) substrates that were designed using a high-resolution electron-beam lithography system (ELS-F125; Elionix, Tokyo, Japan) operating at $125 \mathrm{kV}$. A conventional copolymer resist (ZEP520A; Zeon Chemicals, Louisville, USA) diluted with a ZEP thinner $(1: 1)$ was spin-coated onto the substrate at 1000 r.p.m. for $10 \mathrm{~s}$ and 4000 r.p.m. for $90 \mathrm{~s}$, and it was then prebaked on a hot plate for $2 \mathrm{~min}$ at $150{ }^{\circ} \mathrm{C}$. The electron-beam lithography operated at an electrical current of $3 \mathrm{nA}$. After development, a 2-nm-thick titanium layer was first deposited via sputtering (MPS-4000; ULVAC, Tokyo, Japan) as the adhesive layer, followed by a 40 -nm-thick gold film. The lift-off was performed by successively immersing the sample in anisole, acetone and ultrapure water in an ultrasonic bath. Four differently shaped Au nanoparticle arrays were utilized in this study, namely, nanorods (rectangular), nanodisk-like particles (round), dimers and nanoblocks (square), and were arranged in two-dimensional arrays on the substrates. Field-emission scanning electron microscopy (JSM-6700FT; JEOL, Tokyo, Japan) and Fourier transform infrared (FTIR) spectroscopy (FT/IR-6000TM-M; JASCO, Tokyo, Japan) were used to characterize the morphologies and far-field spectral properties of the $\mathrm{Au}$ nanostructures, respectively.

The photoemission electron microscope used in this study was a PEEM with an energy analyzer (Elmitec $\mathrm{GmbH}$, Clausthal-Zellerfeld, Germany) with a spatial resolution of over $8 \mathrm{~nm}$. As an excitation source, we used either a mercury lamp (unpolarized continuous wave light with a cutoff energy of $4.9 \mathrm{eV}$ ) or a mode-locked Ti:sapphire oscillator (Rainbow; Femtolasers, Vienna, Austria) that delivered a 7 -fs laser pulse at a central wavelength of $800 \mathrm{~nm}$ with a $>200-\mathrm{nm}$ bandwidth at a repetition rate of $77 \mathrm{MHz}$. The sample was irradiated at an angle of $74^{\circ}$. The femtosecond laser beam was focused down to a spot of approximately $50 \mu \mathrm{m} \times 200 \mu \mathrm{m}$ on the sample using a lens (focal length $f=150 \mathrm{~mm}$ ). Chirp mirror pairs and a wedge pair were used to compensate for the dispersion to obtain the shortest pulse duration inside the PEEM chamber. The polarization of the laser beam was controlled by a $\lambda / 2$ waveplate, and a p-polarized beam was used in this study. The interferometric time-resolved setup was based on a Mach-Zehnder interferometer. Typically, time-resolved PEEM images can be recorded by adjusting the delay time at a step corresponding to a $\pi / 2$ phase delay (for a carrier wavelength of $800 \mathrm{~nm}$, is $0.67 \mathrm{fs}$ ).

\section{RESULTS AND DISCUSSION}

The first sample was an $\mathrm{Au}$ nanorod array with a footprint of $95 \mathrm{~nm} \times 180 \mathrm{~nm}$ and a thickness of $40 \mathrm{~nm}$. Figure 1a is an SEM image of the fabricated Au nanorods exhibiting good structural quality and uniformity. The experimental configuration of light irradiation is schematically shown in Figure 1b; either the continuous UV light (from a mercury lamp) or the femtosecond laser pulse was irradiated onto the sample from the left side of Figure $1 \mathrm{c}$ and $1 \mathrm{~d}$ at an incidence angle of $74^{\circ}$ from the surface normal. In the case of the excitation by femtosecond laser pulses, the p-polarized beam was used. The PEEM images of the same array excited via UV light (from a mercury lamp) and the femtosecond laser beam are provided in Figure 1c and 1d, respectively, and demonstrate a striking difference. Specifically, the uniform photoemission from the Au nanorods can be observed in Figure 1c; however, the photoemission is highly localized at the corners of Au nanorods in Figure 1d.

The typical work function for gold is $4.6-5.1 \mathrm{eV}$ depending on the crystal facet, ${ }^{40}$ and the work function of $\mathrm{TiO}_{2}(110)$ is $\sim 5.2 \mathrm{eV}$. $^{41}$ Because the cutoff photon energy of a mercury lamp is $4.9 \mathrm{eV}$, the Au surface can photoemit, while the $\mathrm{TiO}_{2}$ substrate cannot, leading to 

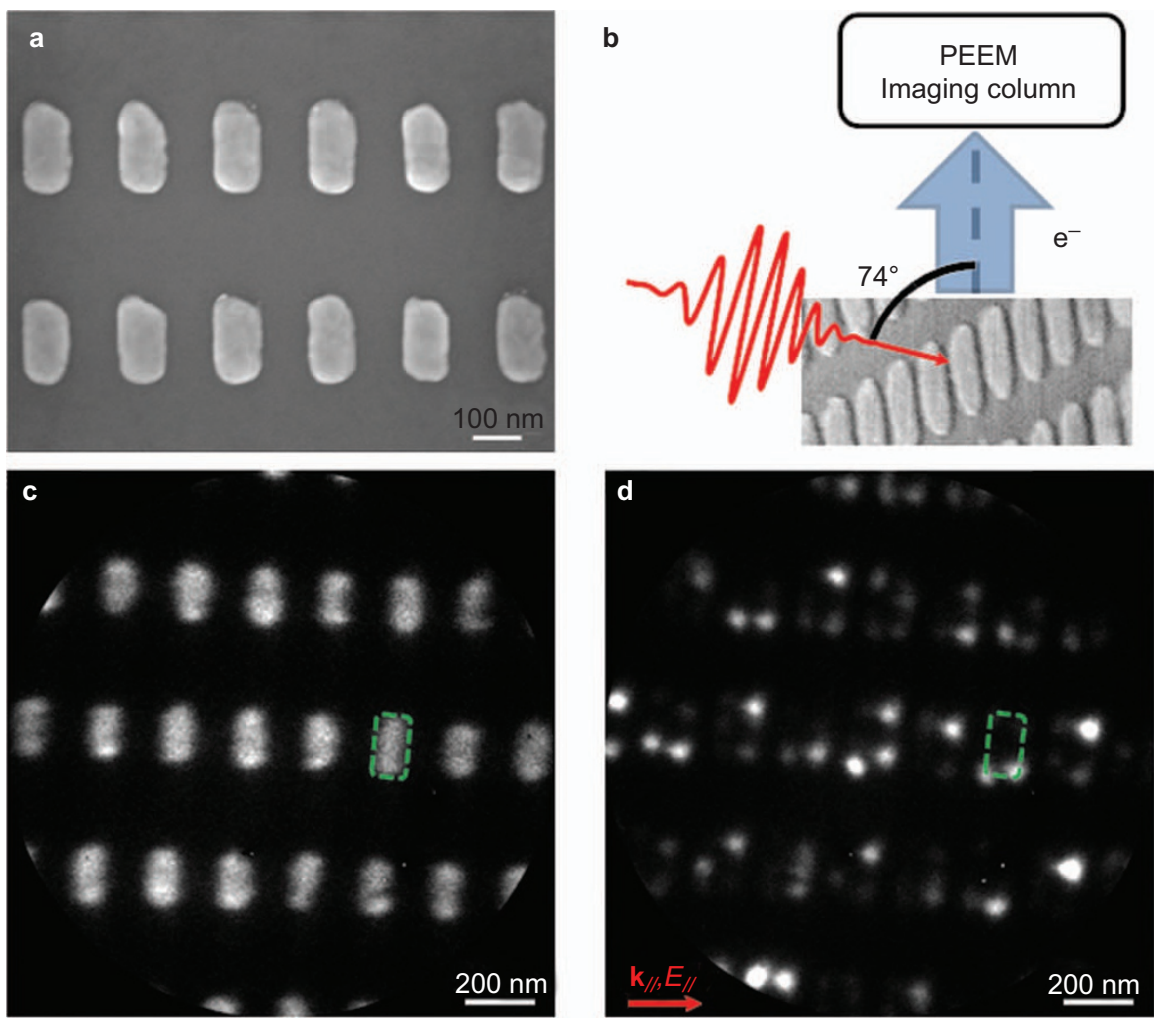

Figure 1 (a) SEM image of an Au nanorod $\left(95 \mathrm{~nm} \times 180 \mathrm{~nm} \times 40 \mathrm{~nm}\right.$ ) array on $\mathrm{Nb}$-doped $\mathrm{TiO}_{2}$. (b) A sketch map of the irradiation setup for the PEEM measurements. PEEM images of the same array excited using (c) a mercury lamp and (d) p-polarized NIR femtosecond laser pulses in which hot spots can be observed at four corners of each rod. A dashed line rectangle in (c) and (d) indicates the outline of an Au nanorod. The light was irradiated from the left side at an incident angle of $74^{\circ}$. The wave vector in the plane of the sample surface $\mathbf{k}_{/ /}$is indicated, and for the p-polarized beam used, the polarization in the plane of the sample surface $E_{/ / /}$is parallel to $\mathbf{k}_{/ /}$. NIR, near-infrared; PEEM, photoemission electron microscopy; SEM, scanning electron microscopy.

the image contrast in Figure $1 c$ that results from the difference in the work function. For the femtosecond laser pulses, a one-photon energy is insufficient to overcome the work function and emit electrons. However, the Au nanorods can support two LSPR modes: one is a longitudinal plasmon mode (L-mode) with the polarization parallel to the long axes of the nanorods, and the other is transverse plasmon mode (T-mode) with the polarization parallel to the short axes of the nanorods. The T-mode of this Au nanorod sample is centered at $740 \mathrm{~nm}$, which overlaps with the laser spectrum as shown in Figure 2a. When the sample is irradiated with p-polarized laser pulses, the T-mode LSPR can be effectively excited. Accordingly, local field enhancement (hot spots) should be expected. The large enhancement of the local field allows multiphoton photoemission. For a resonance wavelength of $740 \mathrm{~nm}(1.70 \mathrm{eV})$, at least three photons are required to induce photoemission, as demonstrated by the laser power-dependent investigation from which a log-log plot of the photoemission vs. the laser power yields a slope of 3.10 (not shown, but a similar example is provided in Figure 3d). Because of the nature of this nonlinear photoemission, the photoemission yield depends on the local electric field intensity to the power of $2 n$ (generally, $n$ is the minimum number of photons required to overcome the work function; in this example, $n$ is 3). The MP-PEEM image in Figure 1d can thus be regarded as the nonlinear mapping of the local field on the gold nanorods, which experimentally confirmed that the near-field intensity is highly localized at particular so-called hot spots.

It is worth noting that the special resolution of particular PEEM images is dependent on many factors, such as the topography of the sample, the light source, the field of view and so on. ${ }^{42}$ In this study, the plasmonic hot spots excited by femtosecond laser pulses are usually located at the corners or edges of the nanostructures. The photoemission from the corners or edges exhibits a large span of the emission angle, which increases the spherical aberration in the imaging column and thus, degrades the spatial resolution a little bit. In addition, the space charge effect may also degrade the spatial resolution. ${ }^{43}$ We tested the real spatial resolution from Figure 1d. It was measured to be 15.0 $( \pm 3.7) \mathrm{nm}$, which was slightly lower than the best spatial resolution $(\sim 8 \mathrm{~nm})$ of our PEEM. An example of a cross section in one of the hot spots in Figure 1d can be found in Supplementary Figure S1), which yields a spatial resolution of $14.4 \mathrm{~nm}$.

To numerically calculate the electromagnetic field distribution around the Au nanorods, we performed finite-difference time-domain (FDTD) simulations (using the FDTD solutions software package from Lumerical, Inc., Vancouver, Canada). In the simulations, the dimensions of nanorods were chosen as the same as the fabricated structures $(95 \mathrm{~nm} \times 180 \mathrm{~nm} \times 40 \mathrm{~nm})$, and the plane wave was incident onto the structures at an incidence angle of $74^{\circ}$, which is identical to the PEEM experiments. The optical properties of the gold were obtained using the data from Johnson and Christy. ${ }^{44}$ The $\mathrm{TiO}_{2}$ substrate was assumed to behave as a dielectric with a constant value for the refractive index $(n=2.6)$. The FDTD simulations were performed on a discrete, non-uniformly spaced mesh with a maximum resolution of $2 \mathrm{~nm}$. Figure $2 \mathrm{~b}$ and $2 \mathrm{c}$ present the electric field intensity distribution at a wavelength of $800 \mathrm{~nm}$, which is the central wavelength of the laser source and within the T-mode resonance range, on the planes located 

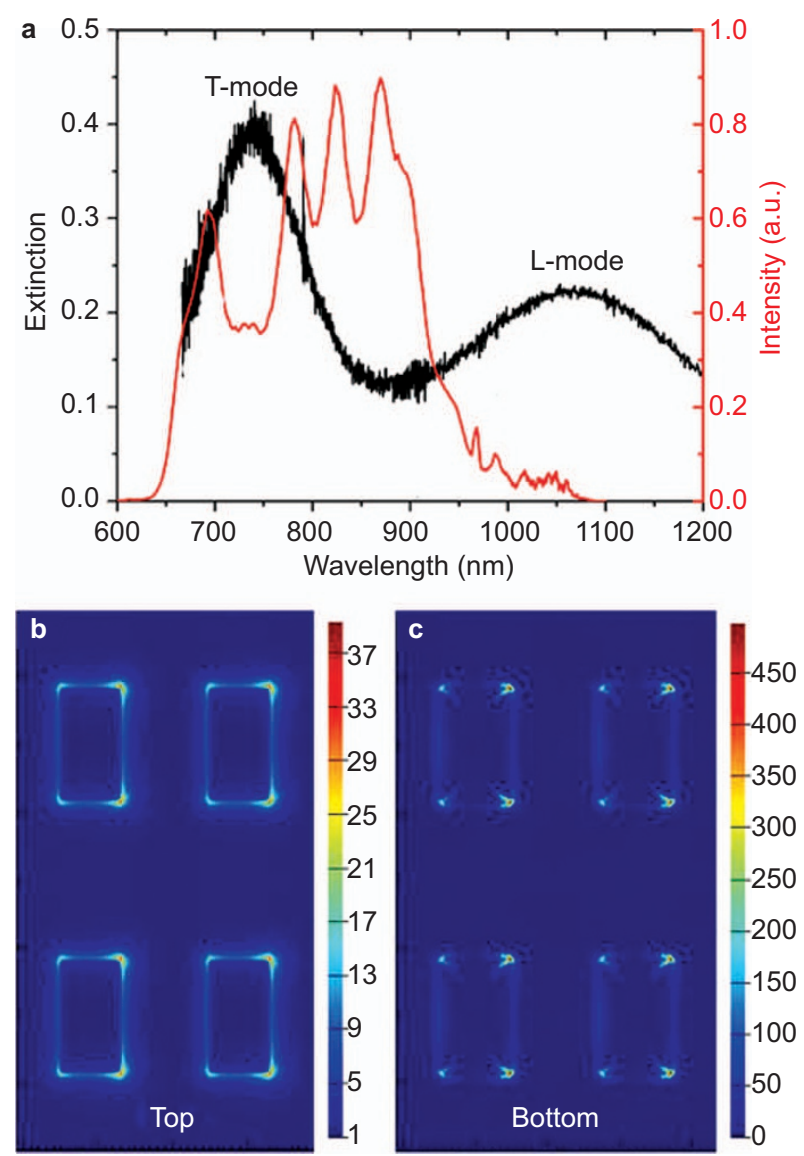

Figure 2 (a) Excitation spectrum (black curve) of the Au nanorods measured via FTIR spectroscopy and the spectrum (red curve) of the femtosecond laser pulse used as the excitation source in the PEEM measurements. The T-mode LSPR at $\sim 740 \mathrm{~nm}$ is overlapped with the laser spectrum. FDTD simulation results for the electric field intensity distribution on the planes at (b) the top and (c) the bottom of the Au nanorods. FDTD, finite-difference time-domain; FTIR, Fourier transform infrared; LSPR, localized surface plasmon resonance; PEEM, photoemission electron microscopy.

at the top and bottom of the Au nanorods, respectively. For both planes, the calculations of the field intensity distribution are in good agreement with the experimental results obtained from the PEEM measurements with the resonant femtosecond laser pulse excitation. The peak enhancement on the bottom plane was much greater than that on the top plane, as predicted by the simulations. However, validating this result using the PEEM measurements is difficult because the photoemissions from different vertical positions are difficult to distinguish. For most nanorods, the hot spots at the right corners of the rods are brighter than those at the left corners. This phenomenon was also well reproduced by the simulations and is attributed to the retardation effect ${ }^{33}$ because the light was irradiated from the left side at a grazing angle. So far, the highly spatially resolved near-field mapping of the LSPR field on the Au nanorods was successfully obtained with the PEEM measurements using femtosecond laser pulse irradiation through nonlinear photoemission.

As previously mentioned, the wavelength and amplitude of the LSPR depend on the shape of the nanostructures. We annealed another $\mathrm{Au}$ nanorod array at $800{ }^{\circ} \mathrm{C}$ in a vacuum. The nanorods transformed into nearly round shape with an average diameter of $135 \mathrm{~nm}$ as seen from the top view of scanning electron microscopy
(SEM) image (Figure 3a). The real shape of the annealed nanoparticles is found to be nanodisk-like shape by the side view of SEM image (shown as Supplementary Figure S2). The L-mode and T-mode LSPR of the nanorods merged into a single mode centered at $800 \mathrm{~nm}$ (Figure 3b), which completely overlapped with the femtosecond laser spectrum. Thus, the LSPR can be efficiently excited. Figure $3 \mathrm{c}$ provides the PEEM image resulting from the irradiation of the sample using $\mathrm{p}$ polarized femtosecond laser pulses. Two hot spots per nanodisk-like particle reside at the two poles along the projected polarization direction on the substrate's surface plane. It is a nature of the dipolar plasmon resonance with the electrons oscillation between the two poles. In this case, the log-log plot of the photoemission yield versus the laser power has a slope of 3.75, indicating that a four-photon process is primarily involved. The nonlinear order is increased compared to the Au nanorod sample investigated above. It is mostly because the LSPR peak within the laser spectra red shifts from $740 \mathrm{~nm}$ to $800 \mathrm{~nm}$ (photon energy at the resonance wavelength becomes lower). In addition, the annealing process may increase the work function of Au. Regarding the mechanism of the nonlinear photoemission, it is also worth noting that field emission is possible as argued in a recent report. ${ }^{45}$ The field emission occurs at the lowfrequency/high-intensity limit of the electric field induced by the electron ejection and is thus referred to as a tunneling emission. The laser intensity used in this study was typically on the order of $10 \mathrm{MW} \mathrm{cm}^{-2}$. Considering the LSPR-induced intensity enhancement factor in the range of 10-1000, the maximum local intensity was only on the order of $10 \mathrm{GW} \mathrm{cm}^{-2}$. Such NIR radiation intensity is not strong enough to trigger field emission. When a large enhancement factor (for example, sharp metal nanotips ${ }^{46,47}$ or small gaps in closely assembled nanostructures $^{25,48,49}$ ) is present, it may enter the field emission region. For the laser intensity typically used in PEEM measurements, both multiphoton photoemission and field emission should be promoted by the local field enhancement effect. Therefore, regardless of the nonlinear photoemission mechanism involved, PEEM is suitable for the near-field mapping of plasmonic nanostructures.

We also examined near-field Au dimer arrays using MP-PEEM. The dimer structures attracted particular interest ${ }^{25,49,50}$ because of the extremely strong enhancement of the electromagnetic field at the nanogap position. Each dimer consists of two diagonally aligned gold nanoblocks with dimensions of approximately $100 \mathrm{~nm} \times 100 \mathrm{~nm} \times$ $40 \mathrm{~nm}$ and a gap size of $30 \mathrm{~nm}$ (an SEM image of a typical dimer can be seen in the inset of Figure 4a). Figure 4a presents the PEEM image resulting from irradiation with femtosecond laser pulses. Two hot spots (one is strong, and the other one is weak) are visible for each dimer, but determining the origin of these hot spots is difficult. To ascertain the positions of the hot spots, the MP-PEEM image normally must be compared with a one-photon PEEM image or an SEM image separately as was done in Figure 1. We demonstrate a simpler and more straightforward method for achieving this goal. Because several ports exist in the main chamber of the photoemission electron microscope, we installed an additional window in one port to allow irradiation from the mercury lamp rather than using the original window designated for the femtosecond laser pulses. Thus, we were able to simultaneously irradiate the sample with UV light and a femtosecond laser. The UV radiation lights up the entire gold dimers through onephoton photoemission, while the multiphoton photoemission yields the localized hot spots. By carefully choosing the light intensities of both irradiation sources, the photoemission from the two different sources can be distinguished. Figure $4 \mathrm{~b}$ provides such a PEEM image in which the sample is simultaneously excited by UV light and 

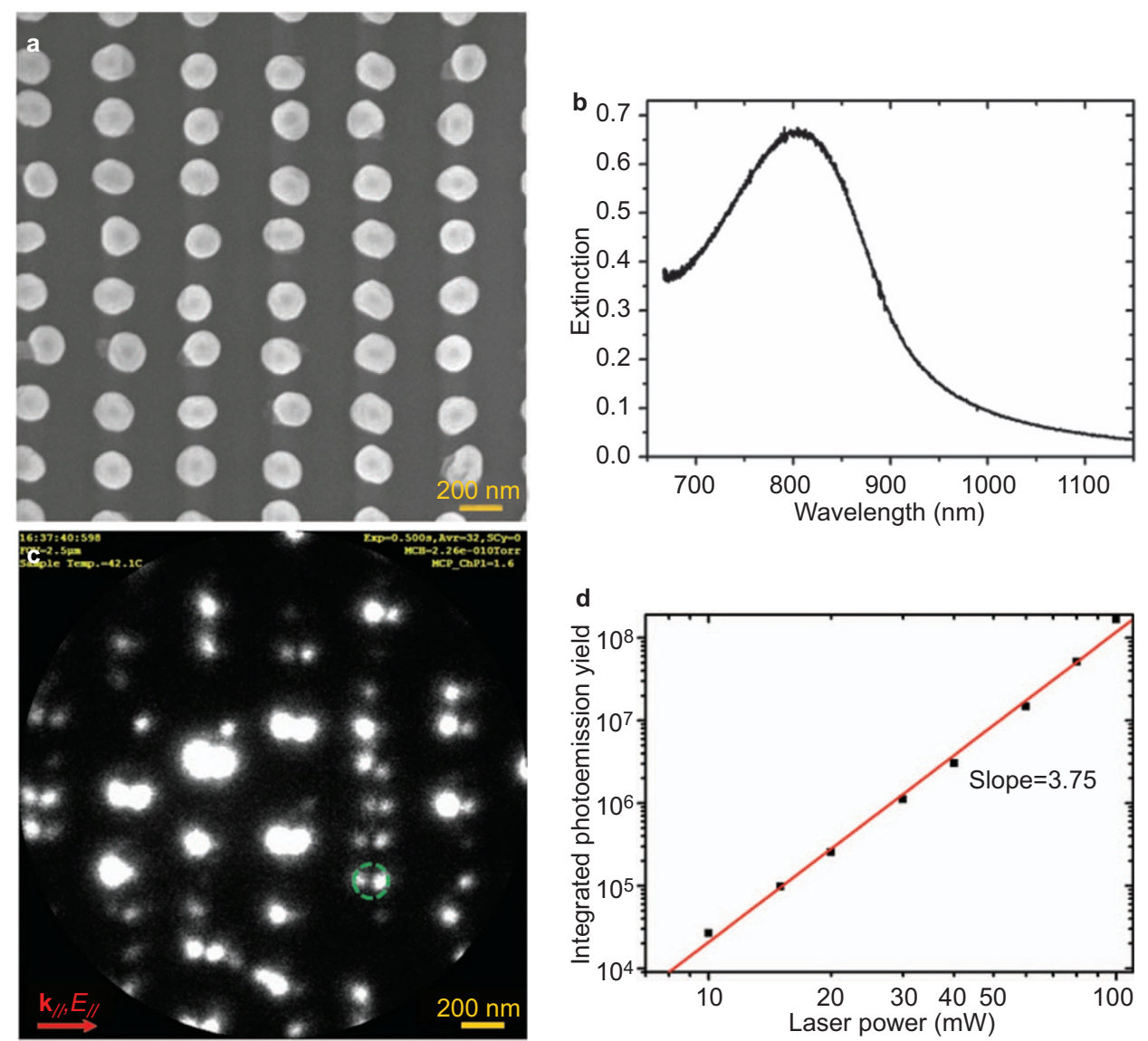

Figure 3 (a) SEM image of the Au nanodisk-like particle array that resulted from the annealing of an Au nanorod sample with an average diameter of $\sim 135 \mathrm{~nm}$. (b) Excitation spectrum of the Au nanoparticles. The single LSPR peak at approximately $800 \mathrm{~nm}$ exactly coincided with the laser spectrum shown in Figure 2a. (c) The MPPEEM image of the Au nanoparticles gives rise to two hot spots at the poles along the laser polarization direction parallel to the substrate surface. A dashed line circle indicates the outline of an Au nanoparticle. (d) Double-logarithmic plot of the integrated photoemission signal versus the laser power yielding a slope of 3.75 in a linear fitting. LSPR, localized surface plasmon resonance; MP-PEEM, multiphoton photoemission electron microscopy; SEM, scanning electron microscopy.

femtosecond laser pulses. The photoemission can be seen across the gold dimers, and the strong hot spot is found at the gap positions. Thus, the strong hot spot from each dimer in Figure 4a should result from the gap position. The weak hot spot of each dimer in Figure 4a is difficult to identify in Figure $4 \mathrm{~b}$ because their intensity is comparable to or even weaker than that from the one-photon photoemission via
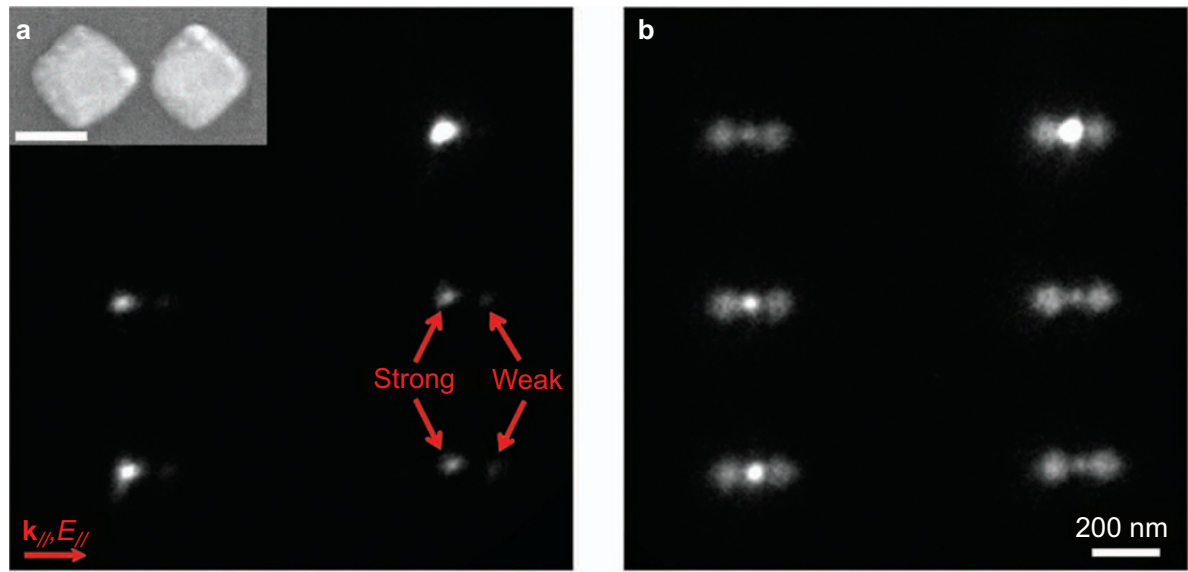

Figure 4 PEEM images of an array of Au dimer structures. (a) An MP-PEEM image showing the irradiation of the NIR femtosecond pulses only. (b) A PEEM image resulting from simultaneous irradiation with the NIR femtosecond pulses and the mercury lamp. The multiphoton photoemission excited by the laser yielded the LSPRassisted hot spots, while the one-photon photoemission by the mercury lamp provided the surface morphology of the gold structures. Thus, the PEEM image (b) allowed the precise determination of the hot spot locations. The inset of (a) provides an SEM image of a single dimer structure; the scale bar is 100 nm. MP-PEEM, multiphoton photoemission electron microscopy; NIR, near-infrared; SEM, scanning electron microscopy. 
UV light. However, we can infer that these hot spots result from the right corners of the right nanoblocks. This finding is consistent with the previous investigations of dimer structures. ${ }^{21,25}$ The nearly complete lack of photoemission from the left corners of the dimers should also result from the retardation effect.

We have demonstrated the application of MP-PEEM for the direct imaging of a near-field LSPR on different Au nanostructure arrays. To investigate the dynamics of the LSPR, we established a TR-MP-PEEM system, which is similar to that used by Petek et al. ${ }^{32,38}$ However, in our experiments, the fundamental beam from the Ti:sapphire femtosecond laser was used instead of its double-frequency beam. As previously stated, the NIR femtosecond pulses are more suitable as the excitation source for studying the LSPR of Au nanostructures. The femtosecond laser pulses pass through a Mach-Zehnder interferometer to form phase-correlated pump-and-probe optical pulse pairs. A series of MP-PEEM images were recorded at a frame interval of $0.67 \mathrm{fs}(\pi / 2 \mathrm{rad}$ with respect to the $800 \mathrm{~nm}$ carrier wave of the laser pulse) by adjusting the delay between the pump and probe pulses. Movies showing the oscillation of the hot spots induced by the LSPR can be made using these frames (an example of a movie recorded for the annealed nanoparticle sample in Figure 3 is provided in Supplementary Movie 01).
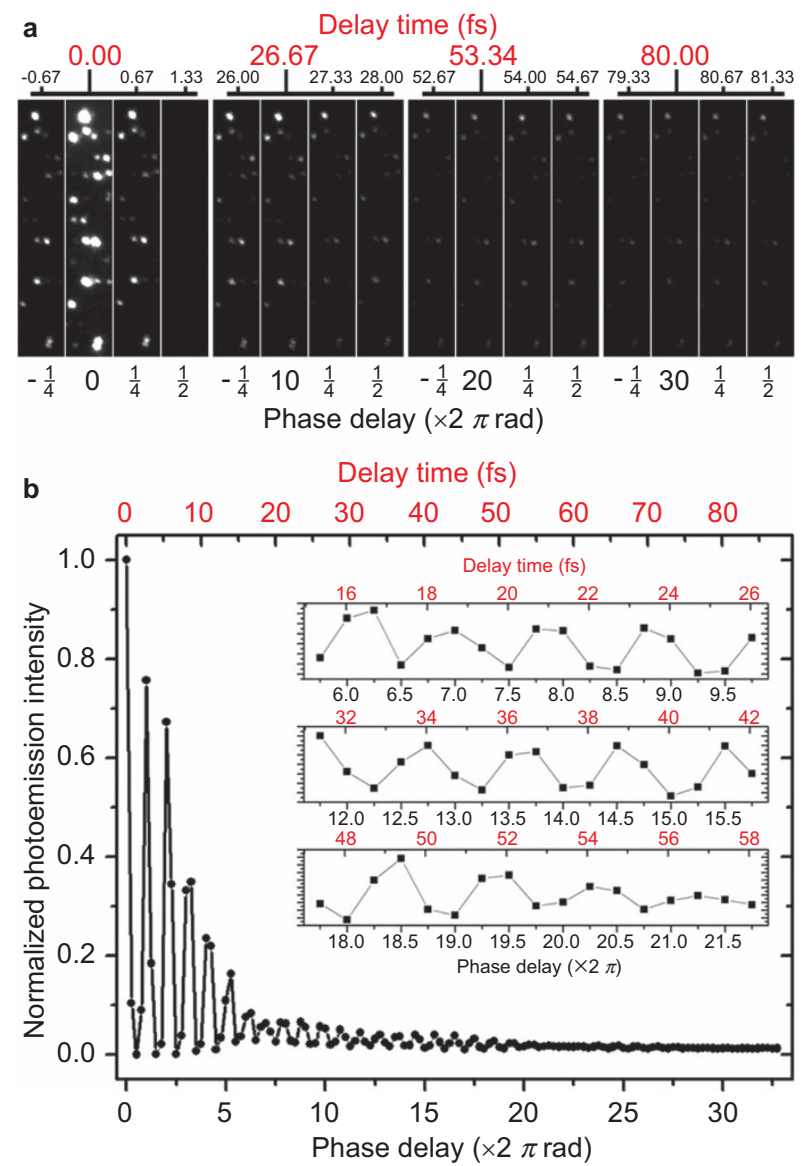

Figure 5 (a) Selected TR-MP-PEEM images at different phase delays/delay times for an array of Au nanorods. (b) Evolution of the photoemission signal with a phase delay/delay time, and several magnified regions are displayed as the insets. TR-MP-PEEM, time-resolved multiphoton photoemission electron microscopy.
Figure 5a presents several images in which the oscillation of SPR fields on Au nanorods can be observed. The evolution of the integrated photoemission signal with the phase delay or delay time is plotted in Figure 5b. In the early stage, the oscillation of the SPR is nearly dominated by the interference of the pump and probe pulses such that it resonates at the laser carrier frequency. When the delay is longer than $15 \mathrm{fs}$, the pump and probe pulses are separate. The coherent LSPR fields excited by the pump pulses can preserve the memory of the optical phase of the excitation pulses; thus, the two LSPR fields induced by the pump and probe pulses can interfere with each other and dominate the oscillation of the hot spots. However, the total photoemission yield decays because of a dephasing of the LSPR field, and the oscillation frequency begins to differ from the carrier frequency. In this region, the LSPR field resonance is at its characteristic frequency and oscillates more rapidly, as shown in the insets of Figure $5 \mathrm{~b}$, because the LSPR wavelength is approximately $740 \mathrm{~nm}$, which is shorter than the carrier wavelength of the excitation pulsed light $(800 \mathrm{~nm})$. At delay times longer than $60 \mathrm{fs}$, almost no oscillation remains because the coherent SP modes have been completely dephased and only weak photoemission signals are induced by the pump and probe beams independently. We should be able to fit out the dephasing time of the LSPR similar to the way in which it was performed in the time-resolved two-photon photoemission from silver nanostructures. ${ }^{32}$ However, performing such a fit is currently difficult because of the problems with real characterizations of the laser pulse incident on the sample in the PEEM chamber and an incomplete understanding of the detailed mechanism of the nonlinear photoemission involved.

To implement the ability of TR-MP-PEEM in imaging the time evolution of the LSPR fields, we prepared a new sample, which consisted of two Au nanoblock arrays of different sizes. The geometry of the two arrays is shown in the inset of Figure 6a. The two Au nanoblock arrays exhibit different LSPR wavelengths. As shown in Figure 6a, which provides reflection spectra with the reflectivity of the bare substrate as a reference, the LSPR is centered at 770 and $830 \mathrm{~nm}$ for array 1 and array 2 , respectively. Figure $6 \mathrm{~b}$ provides the time evolution of the photoemission from both arrays. When the phase delay between the pump and probe beams is less than $3 \times 2 \pi \mathrm{rad}$, the photoemission yield from both arrays oscillates at the carrier frequency of the laser. However, with a phase delay longer than $3 \times 2 \pi \mathrm{rad}$, the hot spots from array 1 begin to oscillate faster than the carrier frequency of the laser and the hot spots from array 2 oscillate slower than the carrier frequency, as clearly presented in the inset of Figure $6 \mathrm{~b}$. This result is due to the hot spot resonance at the characteristic LSPR wavelength for each array as previously discussed. A movie recording the out-of-phase oscillation for the hotspots from these two arrays can be found in Supplementary Movie 02. This result indicates that a small difference in the intrinsic SPR frequencies can be identified via TR-MP-PEEM.

\section{CONCLUSIONS}

In this work, MP-PEEM was employed to investigate the near-field properties of the LSPR on Au nanoparticles, including its near-field distribution and the dynamics excited by ultrashort laser pulses in the NIR region. For three arrays of Au nanoparticles with different shapes (nanorods, nanodisk-like particles and dimers), the near-field mapping is directly obtained as the distribution of the hot spots in the MPPEEM images. In particular, the location of the hot spots can be determined in a precise and straightforward manner by simultaneous excitation with UV light and NIR femtosecond pulses. The ultrafast dynamics of the LSPR were also probed at femtosecond temporal and 
a

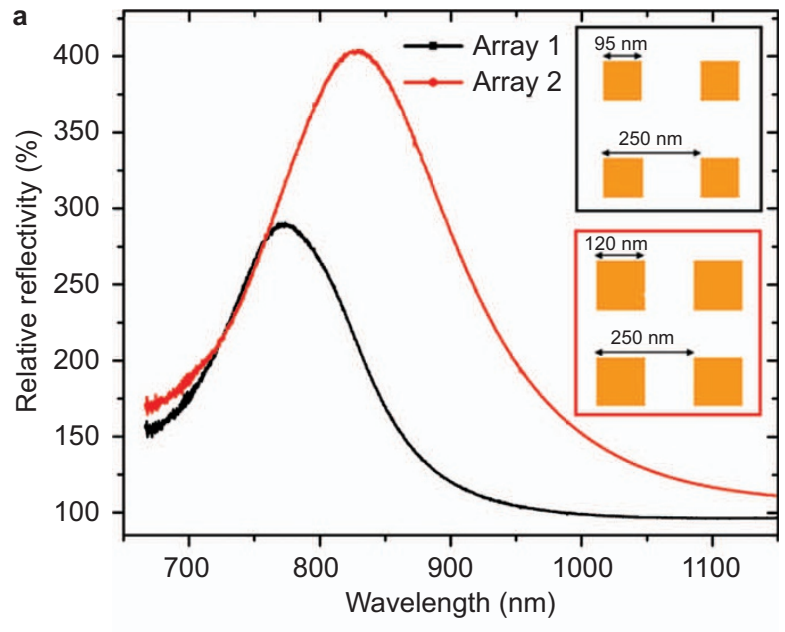

b

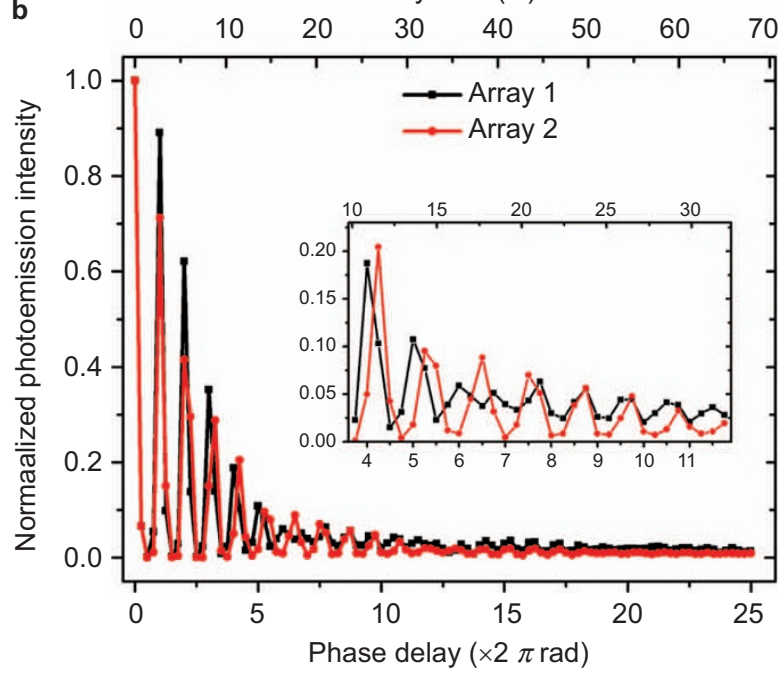

Figure 6 (a) Reflection spectra and (b) the evolution of the photoemission signal with a phase delay via TR-MP-PEEM measurements for the two Au nanoblock arrays whose geometries are provided in the insets of (a). TR-MP-PEEM, timeresolved multiphoton photoemission electron microscopy.

nanometer spatial resolution. To our knowledge, this paper is the first to report a time-resolved study of the LSPR of Au nanoparticles via PEEM that intuitively demonstrates the oscillation and dephasing of an LSPR field in the NIR region. This study will aid in elucidation of the mechanism of LSPR, including the confinement effect and the decay channels, and provides an approach for optimizing the design of plasmonic-based devices for many unique applications.

\section{ACKNOWLEDGMENTS}

The authors would like to thank Dr Youzhuan Zhang and Mr Xu Shi for preparing the samples in the early stage of this study. This study was supported by funding from the Ministry of Education, Culture, Sports, Science, and Technology of Japan: KAKENHI Grant-in-Aid for Scientific Research No. 23225006, Nanotechnology Platform (Hokkaido University) and the Low-Carbon Research Network of Japan.

1 Jain PK, Huang XH, El-Sayed IH, El-Sayed MA. Noble metals on the nanoscale: optical and photothermal properties and some applications in imaging, sensing, biology, and medicine. Accounts Chem Res 2008; 41: 1578-1586.
2 Giannini V, Fernandez-Dominguez Al, Sonnefraud Y, Roschuk T, Fernandez-Garcia R et al. Controlling light localization and light-matter interactions with nanoplasmonics. Small 2010; 6: 2498-2507.

3 Schuller JA, Barnard ES, Cai WS, Jun YC, White JS et al. Plasmonics for extreme light concentration and manipulation. Nat Mater 2010; 9: 193-204.

4 Watanabe K, Menzel D, Nilius N, Freund HJ. Photochemistry on metal nanoparticles. Chem Rev 2006; 106: 4301-4320.

5 Chen C, Hutchison JA, Clemente F, Kox R, Uji-I H et al. Direct evidence of high spatial localization of hot spots in surface-enhanced Raman scattering. Angew Chem Int Ed 2009; 48: 9932-9935.

6 Yokota Y, Ueno K, Misawa H. Highly controlled surface-enhanced raman scattering chips using nanoengineered gold blocks. Small 2011; 7: 252-258.

7 Alvarez-Puebla RA, Agarwal A, Manna P, Khanal BP, Aldeanueva-Potel P et al. Gold nanorods 3D-supercrystals as surface enhanced Raman scattering spectroscopy substrates for the rapid detection of scrambled prions. Proc Natl Acad Sci USA 2011; 108: 8157-8161.

8 Hutchison JA, Centeno SP, Odaka H, Fukumura H, Hofkens J et al. Subdiffraction limited, remote excitation of surface enhanced Raman scattering. Nano Lett2009; 9: 995-1001.

9 Nusz GJ, Curry AC, Marinakos SM, Wax A, Chilkoti A. Rational selection of gold nanorod geometry for label-free plasmonic biosensors. ACS Nano 2009; 3: 795-806.

10 Lee KL, Chih MJ, Shi X, Ueno K, Misawa H et al. Improving surface plasmon detection in gold nanostructures using a multi-polarization spectral integration method. Adv Mater 2012; 24: 253-259.

11 Wu BT, Ueno K, Yokota Y, Sun K, Zeng HP et al. Enhancement of a two-photoninduced reaction in solution using light-harvesting gold nanodimer structures. J Phys Chem Lett 2012; 3: 1443-1447.

12 Tsuboi Y, Shimizu R, Shoji T, Kitamura N. Near-infrared continuous-wave light driving a two-photon photochromic reaction with the assistance of localized surface plasmon. J Am Chem Soc 2009; 131: 12623-12627.

13 Nishijima Y, Ueno K, Yokota Y, Murakoshi K, Misawa H. Plasmon-assisted photocurrent generation from visible to near-infrared wavelength using a Aunanorods/TiO 2 electrode. J Phys Chem Lett 2010; 1: 2031-2036.

14 Sugawa K, Akiyama T, Kawazumi H, Yamada S. Plasmon-enhanced photocurrent generation from self-assembled monolayers of phthalocyanine by using gold nanoparticle films. Langmuir 2009; 25: 3887-3893.

15 Su YH, Ke YF, Cai SL, Yao QY. Surface plasmon resonance of layer-by-layer gold nanoparticles induced photoelectric current in environmentally-friendly plasmonsensitized solar cell. Light Sci App/ 2012; 1: e14.

16 Grubisic A, Ringe E, Cobley CM, Xia YN, Marks LD et al. Plasmonic near-electric field enhancement effects in ultrafast photoelectron emission: correlated spatial and laser polarization microscopy studies of individual Ag nanocubes. Nano Lett 2012; 12: 4823-4829.

17 Schweikhard V, Grubisic A, Baker TA, Thomann I, Nesbitt DJ. Polarization-dependent scanning photoionization microscopy: ultrafast plasmon-mediated electron ejection dynamics in single Au nanorods. ACS Nano 2011; 5: 3724-3735.

18 Imura K, Nagahara T, Okamoto H. Near-field optical imaging of plasmon modes in gold nanorods. J Chem Phys 2005; 122: 154701

19 Imura K, Ueno K, Misawa H, Okamoto H. Anomalous light transmission from plasmonic-capped nanoapertures. Nano Lett 2011; 11: 960-965.

20 Rang M, Jones AC, Zhou F, Li ZY, Wiley BJ et al. Optical near-field mapping of plasmonic nanoprisms. Nano Lett 2008; 8: 3357-3363.

21 Tanaka Y, Ishiguro H, Fujiwara H, Yokota Y, Ueno K et al. Direct imaging of nanogapmode plasmon-resonant fields. Opt Express 2011; 19: 7726-7733.

22 Ueno K, Juodkazis S, Mizeikis V, Sasaki K, Misawa H. Clusters of closely spaced gold nanoparticles as a source of two-photon photoluminescence at visible wavelengths. Adv Mater 2008; 20: 26-30.

23 Imura K, Okamoto H, Hossain MK, Kitajima M. Visualization of localized intense optical fields in single gold-nanoparticle assemblies and ultrasensitive Raman active sites. Nano Lett 2006; 6: 2173-2176.

24 Geldhauser T, Kolloch A, Murazawa N, Ueno K, Boneberg J et al. Quantitative measurement of the near-field enhancement of nanostructures by two-photon polymerization. Langmuir 2012; 28: 9041-9046.

25 Ueno K, Juodkazis S, Shibuya T, Yokota Y, Mizeikis V et al. Nanoparticle plasmonassisted two-photon polymerization induced by incoherent excitation source. J Am Chem Soc 2008; 130: 6928-6929.

26 Leiderer P, Bartels C, Konig-Birk J, Mosbacher M, Boneberg J. Imaging optical nearfields of nanostructures. Appl Phys Lett 2004; 85: 5370-5372.

27 Nedyalkov NN, Takada H, Obara M. Nanostructuring of silicon surface by femtosecond laser pulse mediated with enhanced near-field of gold nanoparticles. Appl Phys $A$ 2006; 85: 163-168.

28 Eversole D, Luk'yanchuk B, Ben-Yakar A. Plasmonic laser nanoablation of silicon by the scattering of femtosecond pulses near gold nanospheres. Appl Phys A 2007; 89: 283-291.

29 Kolloch A, Geldhauser T, Ueno K, Misawa H, Boneberg J et al. Femtosecond and picosecond near-field ablation of gold nanotriangles: nanostructuring and nanomelting. App/ Phys A 2011; 104: 793-799.

30 Cinchetti M, Gloskovskii A, Nepjiko SA, Schonhense G, Rochholz $\mathrm{H}$ et al. Photoemission electron microscopy as a tool for the investigation of optical near fields. Phys Rev Lett 2005; 95: 047601.

31 Heringdorf FM, Chelaru LI, Mollenbeck S, Thien D, Hoegen MH. Femtosecond photoemission microscopy. Surf Sci 2007; 601: 4700-4705. 
32 Kubo A, Onda K, Petek H, Sun ZJ, Jung YS et al. Femtosecond imaging of surface plasmon dynamics in a nanostructured silver film. Nano Lett 2005; 5: 1123-1127.

33 Melchior P, Bayer D, Schneider C, Fischer A, Rohmer M et al. Optical near-field interference in the excitation of a bowtie nanoantenna. Phys Rev B 2011; 83: 235407.

34 Schertz F, Schmelzeisen M, Mohammadi R, Kreiter M, Elmers HJ et al. Near field of strongly coupled plasmons: uncovering dark modes. Nano Lett 2012; 12: 1885-1890.

35 Aeschlimann M, Brixner T, Fischer A, Kramer C, Melchior P et al. Coherent twodimensional nanoscopy. Science 2011; 333: 1723-1726.

36 Bauer E, Mundschau M, Swiech W, Telieps W. Surface studies by low-energy electronmicroscopy (LEEM) and conventional UV photoemission electron-microscopy (PEEM). Ultramicroscopy 1989; 31: 49-57.

37 Vogel J, Kuch W, Bonfim M, Camarero J, Pennec $Y$ et al. Time-resolved magnetic domain imaging by X-ray photoemission electron microscopy. Appl Phys Lett 2003; 82: 2299-2301.

38 Kubo A, Pontius N, Petek H. Femtosecond microscopy of surface plasmon polariton wave packet evolution at the silver/vacuum interface. Nano Lett 2007; 7: 470-475.

39 Zhang LX, Kubo A, Wang LM, Petek H, Seideman T. Imaging of surface plasmon polariton fields excited at a nanometer-scale slit. Phys Rev B 2011; 84: 245442.

40 Michaelson HB. Work function of elements and its periodicity. J Appl Phys 1977; 48: 4729-4733.

41 Schierbaum KD, Fischer S, Torquemada MC, deSegovia JL, Roman E et al. The interaction of Pt with $\mathrm{TiO}_{2}$ (110) surfaces: a comparative XPS, UPS, ISS, and ESD study. Surf Sci 1996; 345: 261-273.

42 Schneider CM, Schonhense G. Investigating surface magnetism by means of photoexcitation electron emission microscopy. Rep Prog Phys 2002; 65: R1785-R1839.
43 Buckanie NM, Gohre J, Zhou P, von der Linde D, Horn-von Hoegen M et al. Space charge effects in photoemission electron microscopy using amplified femtosecond laser pulses. J Phys Condens Matter 2009; 21: 314003.

44 Johnson PB, Christy RW. Optical constants of noble metals. Phys Rev B 1972; 6 : 4370-4379.

45 Schertz F, Schmelzeisen M, Kreiter M, Elmers HJ, Schonhense G. Field emission of electrons generated by the near field of strongly coupled plasmons. Phys Rev Lett 2012; 108: 237602.

46 Bormann R, Gulde M, Weismann A, Yalunin SV, Ropers C. Tip-enhanced strong-field photoemission. Phys Rev Lett 2010; 105: 147601.

47 Kruger M, Schenk M, Forster M, Hommelhoff P. Attosecond physics in photoemission from a metal nanotip. J Phys B At Mol Opt 2012; 45: 074006.

48 Ward DR, Huser F, Pauly F, Cuevas JC, Natelson D. Optical rectification and field enhancement in a plasmonic nanogap. Nat Nanotechnol 2010; 5: 732-736.

49 Hao E, Schatz GC. Electromagnetic fields around silver nanoparticles and dimers. J Chem Phys 2004; 120: 357-366.

50 Acimovic SS, Kreuzer MP, Gonzalez MU, Quidant R. Plasmon near-field coupling in metal dimers as a step toward single-molecule sensing. ACS Nano 2009; 3: 12311237.

(c) (i) (2) This work is licensed under a Creative Commons Attribution-

BY NA NonCommercial-ShareAlike 3.0 Unported license. To view a copy of this

license, visit http://creativecommons.org/licenses/by-nc-sa/3.0

Supplementary Information for this article can be found on Light: Science \& Applications' website (http://www.nature.com/lsa/). 